\title{
A novel identified circular RNA, circ_0000491, aggravates the extracellular matrix of diabetic nephropathy glomerular mesangial cells through suppressing miR-101b by targeting TGF $\beta R I$
}

\author{
XIN MOU*, JIA WEI CHEN*, DI YI ZHOU, KAIYUAN LIU, LI JUN CHEN, DANYANG ZHOU and YONG BIN HU \\ Department of Endocrinology, Zhejiang Integrated and Western Medicine Hospital, Hangzhou, Zhejiang 310003, P.R. China
}

Received August 6, 2019; Accepted August 6, 2020

DOI: $10.3892 / \mathrm{mmr} .2020 .11486$

\begin{abstract}
Circular RNAs (circRNAs) have crucial roles in various diseases; however, the mechanisms of action underlying circRNAs in the occurrence and development of diabetic nephropathy (DN) remains largely unknown. The present study investigated the differentially expressed circRNAs in the DN mice kidney cortex using circRNA sequencing and elucidated the role of circRNAs in mesangial cells. It was revealed that 40 circRNAs were unconventionally expressed, including 18 upregulated circRNAs and 22 downregulated circRNAs. Furthermore, circ_0000491 levels were significantly augmented in both DN mice and high glucose (HG, $30 \mathrm{mM}$ )-induced mouse mesangial cells (MES13 cells). Knockdown of circ_0000491 significantly suppressed the increase of vimentin, fibronectin and $\alpha$-smooth muscle actin, as well as collagen type I, III and IV, whilst reversing the decrease of E-cadherin in HG-induced MES13 cells. It was further revealed that circRNA_0000491 sponged miR-101b and that miR-101b directly targets TGF $\beta$ RI. In addition, the expression levels of miR-101b were negatively associated with the transcriptional level of circRNA_0000491 and miR-101b inhibitors reversed the suppression of extracellular matrix (ECM)-associated protein synthesis mediated by knocking-down circRNA_0000491. In conclusion, the present study investigated the circRNA_0000491/miR-101b/TGFßRI axis in ECM accumulation and fibrosis-associated protein expression levels of mesangial cells, which suggested that circRNA_0000491 may be beneficial for the development of an effective therapeutic target for DN.
\end{abstract}

Correspondence to: Professor Yong Bin Hu, Department of Endocrinology, Zhejiang Integrated and Western Medicine Hospital, 208 East Road, Hangzhou, Zhejiang 310003, P.R. China

E-mail: ybhiwmh@163.com

*Contributed equally

Key words: diabetic nephropathies, MES13 cells, circular RNA, extracellular matrix, renal fibrosis

\section{Introduction}

Diabetic nephropathy (DN) is becoming the most pervasive complication for both types I and II diabetes, and is also the major cause of end-stage renal disease in the majority of developed and developing countries (1). Clinically, DN is characterized by mesangial hypertrophy, mesangial cell (MC) proliferation, albuminuria, extracellular matrix (ECM) accumulation and kidney fibrosis $(2,3)$. It has been recognized that the glomerular MCs and ECM accumulation are involved in a variety of biological events (4). A recent study suggested that high concentration glucose-induced MCs contribute to the initiation and development of kidney fibrosis with an elevated level of fibroin and cytokines expression (5). Thus, it is of utmost importance to investigate the key targets and mechanisms of action underlying the action of MCs in high glucose for the treatment and prevention strategies of DN.

Studies have also begun to demonstrate the participation and regulatory mechanism of non-coding RNAs (ncRNAs) on DN development (6,7). Circular RNAs (circRNAs) are a novel class of endogenous ncRNAs, which mediate diverse physiological and pathological changes in the human body (8), including DN, and are characterized by a covalently-closed loop formed by back-splicing between the $5^{\prime}$ to $3^{\prime}$ ends of the polyA tail $(9,10)$. Initially, circRNAs were incorrectly recognized as splicing errors without any biological functions. There is an increasing evidence indicating that some circRNAs play a significant role in regulating various human diseases through a competing endogenous RNA (ceRNA) mechanism, also known as a micro (mi)RNA sponge $(5,11)$. For example, Li et al (12) revealed that overexpression of circRNA circ-0001785 promotes the proliferative ability of osteosarcoma cells, acting as a ceRNA by sponging miR-1200 to upregulate HOXB2. circ homeodomain interacting protein kinase 3 (HIPK3) is upregulated in Ang II-induced cardiac fibroblasts (CFs) and heart tissues, promoting the proliferation and migration of CFs by acting as a miR-29b-3p sponge (13), suggesting that circRNAs may accelerate the development of fibrosis by acting as an miRNA sponge. Additionally, Chen et al (1) have shown that the circ LDL receptor related protein 6 (LRP6) acts as a 'sponge' of miR-205 to promote ECM-related protein synthesis in MCs by upregulating high 
mobility group box 1 (HMGB1) and activating Toll-like receptor 4 (TLR4)/NF- $\kappa B$ pathway. Similarly, a study by Hu et al (5) found that the circRNA_15698/miR-185/TGF- $\beta 1$ signaling pathway promoted ECM accumulation in DN MCs. Although advances have been made into the roles of circRNAs in human diseases, the role of more novel circRNAs on ECM accumulation and fibrosis-associated proteins, as well as the detailed mechanisms of actions that circRNAs play in MCs remains unclear.

The present study demonstrated a high expression level of mmu_circRNA_0000491 (chr13:94111710-94126034) in mice with DN, as well as the MCs that received high concentration glucose treatment. In addition, the present study also investigated the involvement of the circRNA_0000491/miR-101b/TGF $\beta$-receptor type I (TGF $\beta R I$ ) axis on the ECM of DN tissue. It was hypothesized that circRNA_0000491 worsens the accumulation of ECM of MCs through negatively suppressing miR-101b by targeting TGF $\beta$ RI.

\section{Materials and methods}

Animals. A total of three six-week-old spontaneous male diabetic $d b / d b$ mice (weight: $\sim 18.3-20 \mathrm{~g}$ ) and three age-matched control littermate $d b / m$ mice (weight: $\sim 17.6-21.4 \mathrm{~g}$ ) were obtained from the Shanghai SLAC Laboratory Animal Co., Ltd. All mice were housed in a pathogen-free facility under a controlled temperature of $22 \pm 1^{\circ} \mathrm{C}, 60 \pm 5 \%$ humidity and a 12-h light and dark cycle, with free access to water and a regular standard diet. All the procedures were approved by the Animal Care and Welfare Committee of Zhejiang Chinese Medical University (approval no. ZSLL-2018-192).

circRNA sequencing. The $d b / m$ control and $d b / d b$ mice were euthanized by cervical dislocation, then the kidney cortex from the renal tissues was isolated. Subsequently, total RNA was extracted from the kidney cortex using TRIzol ${ }^{\circledR}$ reagent (Invitrogen; Thermo Fisher Scientific, Inc.), and RNA purity, concentration and integrity were measured using a NanoPhotometer ${ }^{\circledR}$ spectrophotometer (IMPLEN), a Qubit $^{\circledR}$ RNA assay kit in the Qubit ${ }^{\circledR}$ 2.0 Flurometer (Thermo Fisher Scientific, Inc.) and an RNA Nano 6000 Assay kit in the Bioanalyzer 2100 system (Agilent Technologies, Inc.), respectively. circRNA microarray analysis was performed by Novogene Co., Ltd. Briefly, a total of $5 \mu \mathrm{g}$ RNA per sample was applied as input material for the RNA sample preparations. Firstly, ribosomal (r)RNA was removed using the Epicentre Ribo-zero ${ }^{\mathrm{TM}}$ rRNA Removal kit (Epicentre; Illumina, Inc.) and rRNA free residue was cleaned up by ethanol precipitation. Subsequently, the linear RNA was digested with 3 units of RNase R (Epicentre; Illumina, Inc.) per $\mu \mathrm{g}$ of RNA. The sequencing libraries were generated with a NEBNext ${ }^{\circledR}$ Ultra $^{\mathrm{TM}}$ Directional RNA Library Prep kit for Illumina ${ }^{\circledR}$ (New England BioLabs, Inc.) according to the manufacturer's protocol. circRNA was detected and identified using find_circ (14) and CIRI2 (15). The differentially expressed circRNAs were identified by utilizing the edgeR $\mathrm{R}$ package (v3.31.4; http://bioconductor.org) in $\mathrm{R}$ (v3.6.1; https://www.r-project.org/) with the threshold set at a $\mathrm{P}<0.01$ and $\mid \log 2$ (fold change) $\mid>1$. To investigate the underlying function of candidate circRNAs, UCSC Genome Browser (http://genome.ucsc.edu/) was used to illustrate their composition (16).

Cell culture and transfection. MES13 cells from a SV40 transgenic mouse (SV40 MES13) were acquired from the Shanghai Academy of Life Sciences, and maintained in DMEM (Sigma-Aldrich; Merck KGaA) supplemented with 10\% FBS (Thermo Fisher Scientific, Inc.) and 1\% penicillin and streptomycin (Welgene Inc.) in a $37^{\circ} \mathrm{C}$ incubator with $5 \% \mathrm{CO}_{2}$. MES13 cells were stimulated with $5.5 \mathrm{mM}$ D-glucose for the control group. For the model group, the MES13 cells were stimulated with $30 \mathrm{mM}$ glucose for $48 \mathrm{~h}$ in order to simulate the condition of the DN cells. In addition, small interfering RNAs (siRNAs), scrambled siRNA (si_Circ_Control), miR-101b mimics/inhibitor and negative controls, used for cell transfections, were purchased from RiboBio Co., Ltd. The sequences used were as follows: CircRNA_0000491 si_Circ_1, 5'-ATATGGACC AAGAATACCAAA-3'; circRNA_0000491 si_Circ-2, 5'-TAT ATGGACCAAGAATACCAA-3'; si_Circ_Control, 5'-TTC TCCGAACGTGTCACGTTT-3'; miR-101b mimic, 5'-GUA CAGUACUGUGAUAGCU-3'; miR-101b inhibitor, 5'-AGC UAUCACAGUACUGUAC-3'. All the specialized siRNAs were transfected into MES13 cells using Lipofectamine ${ }^{\circledR} 3000$ (Gibco; Thermo Fisher Scientific, Inc.). The subsequent experiments were performed in MES13 cells following $48 \mathrm{~h}$ transfection.

Reverse transcription-quantitative ( $R T-q) P C R$. Total RNA was obtained from kidney samples and MES13 cells using TRIzol ${ }^{\circledast}$ reagent (Invitrogen; Thermo Fisher Scientific, Inc.) and reverse transcribed into cDNA using a PrimeScript RT reagent kit (Takara Bio, Inc.). The reverse transcription conditions were $37^{\circ} \mathrm{C}$ for $15 \mathrm{~min}$, and reverse transcriptase inactivation at $85^{\circ} \mathrm{C}$ for $5 \mathrm{sec}$. For miRNA quantification, cDNA synthesis was performed using the Mir- ${ }^{\mathrm{TM}}$ miRNA First-Strand Synthesis kit (Clontech Laboratories, Inc.). Subsequently, qPCR was performed using a CFX96 Real-Time PCR Detection System (Bio-Rad Laboratories, Inc.) with the amplification conditions: Pre-denaturation at $95^{\circ} \mathrm{C}$ for $30 \mathrm{sec}$, followed by 40 cycles of denaturation at $95^{\circ} \mathrm{C}$ for $5 \mathrm{sec}$ and annealing at $60^{\circ} \mathrm{C}$ for $30 \mathrm{sec}$. The dissolution curve conditions were $65^{\circ} \mathrm{C}$ for $0.05 \mathrm{sec}$ and $95^{\circ} \mathrm{C}$ for $0.5 \mathrm{sec}$. The primers used for RT-qPCR were as follows: mmu_circ_0000491 forward, 5'-TCGGCTTGCAAAGGAGAAGTC-3' and reverse, 5'-TGC ATGCTTGCTGGTGGGTA-3'; E-cadherin (E-cad) forward, 5'-CTCAAGCTCGCGGATAACCA-3' and reverse, 5'-AAT CCTGCTGCCACGATTC-3'; vimentin (Vim) forward, 5'-TGTGACAACTGCCGTAGACC-3' and reverse, 5'-TAG CGGCATGAAGCACTCAA-3'; Fibronectin (FN) forward, 5'-AAGAAGGGCTCGTGTGACAG-3' and reverse, 5'-GGA AGGGTTACCAGTTGGGG-3' ; $\alpha$-SMA forward, 5'-AAG AGAGGGATCCTGACGCT-3' and reverse, 5'-CTCCAG GGAGGAAGAGGAGG-3'; Type I collagen (Col. I) forward, 5'-CGTATCACCAAACTCAGAAGATGT-3', and reverse, 5'-AGAGCCTCTAGCTCCTTGGG-3'; Type III collagen (Col. III) forward, 5'-ATGCCCACAGCCTTCTACAC-3' and reverse, 5'-GGGTCACCATTTCTCCCAGG-3'; Type IV collagen (Col. IV) forward, 5'-GGTGTTCCAGGAAGA GGCTT-3' and reverse, 5'-CATGCCTTGGAATCCTGG GT-3'; TGF $\beta$ RI forward, 5'-AGCTCCTCATCGTGTTGG 
TG-3' and reverse, 5'-AAACCGACCTTTGCCAATGC-3'; miR-101b forward, 5'-GGGCTACTGTGATAGCTAAAA-3'; and 5'-CCAGTGCAGGGTCCGAGGTA-3', as the reverse. In addition, the transcriptional levels of mmu_circ_0000712, mmu_circ_0000898, novel_circ_0001857 and novel_ circ_0001778 were estimated in the kidney samples of $d b / d \bar{b}$ mice. The sequences and primers of mmu_circ_0000712, mmu_circ_0000898, novel_circ_0001857 and novel_ circ_0001778 are shown in Tables SI and SII. GAPDH was used as internal control for the circRNA and mRNA. U6 acted as an internal standard of miR-101b. Relative quantification of expression was performed compared with internal standard with the $2^{-\Delta \Delta \mathrm{Cq}}$ method (17).

Western blotting. Total cellular protein samples were acquired at $4^{\circ} \mathrm{C}$ in RIPA buffer (Sigma-Aldrich; Merck KGaA), then the protein concentration was estimated using a BCA kit (Beyotime Institute of Biotechnology). Proteins ( $40 \mu \mathrm{g})$ were separated by SDS-PAGE (10-12\% gel) and transferred onto polyvinylidene fluoride membranes (GE Healthcare). The membranes were blocked with 5\% skim milk powder for $2 \mathrm{~h}$ at room temperature, and incubated overnight at $4^{\circ} \mathrm{C}$ with primary antibodies against E-cadherin (1:1,000, cat. no. 14472, Cell Signaling Technology, Inc.), vimentin (1:1,000, cat. no. 5741, Cell Signaling Technology, Inc.), FN (1:1,000, ab268020, Abcam), $\alpha$-SMA (1:1,000, cat. no. 68463 , Cell Signaling Technology, Inc.), Type I collagen $(1: 1,000$, cat. no. 39952 , Cell Signaling Technology, Inc.), Type III collagen (1:1,000, ab184993, Abcam), Type IV collagen (1:1,000, cat. no. 50273, Cell Signaling Technology, Inc.), TGF $\beta 1$ (1:1,000, sc-130348, Santa Cruz Biotechnology, Inc.), TGFßR1 (1:2,000, ab31013, Abcam), phosphorylated (p-)Smad3 (1:2,000, ab52903, Abcam), and Smad3 (1:2,000, ab40854, Abcam), with GAPDH serving as an internal control (1:1,000, D190090-0200, Sangon Biotech Co., Ltd.). After exposure to horseradish peroxidase (HRP)-labeled goat anti-rabbit (cat. no. A24531, 1:5,000, Invitrogen; Thermo Fisher Scientific, Inc.) or goat anti-mouse secondary antibodies (cat. no. 31432, 1:5,000, Invitrogen; Thermo Fisher Scientific, Inc.) at room temperature for $2 \mathrm{~h}$, the bands were visualized with enhanced chemiluminescence (Beyotime Institute of Biotechnology) and analyzed using an Odyssey ${ }^{\circledR}$ IR scanner (LI-COR Biosciences).

Luciferase reporter assay. Using the starBase v3.0 database (http://starbase.sysu.edu.cn/index.php), the interactions between circ_0000491 and miR-101b were confirmed. starBase is an open-source platform for studying the miRNA-ncRNA interactions from CLIP-seq, degradome-seq and RNA-RNA interactome data (18). In addition, the mRNAs targeted by miR-101b were retrieved from the TargetScan (version 7.2, http://www.targetscan.org/vert_72/) (19), miRDB (http://mirdb.org/) (20) and PicTar(https://pictar. mdc-berlin.de/) databases. Then, the luciferase reporter assay was applied in MES13 cells. Briefly, MES13 cells were plated into 24-well plates at 60\% confluence. circ_0000491/TGFßRI reporter construct (mutant or wild-type) or the empty reporter vector psiCHECK-2 (Promega Corporation) was co-transfected with miR-101b mimics or control mimics using Lipofectamine ${ }^{\circledR} 3000$ (Thermo Fisher Scientific, Inc.). After $48 \mathrm{~h}$ of transfection, the luciferase activity was measured with a Dual-Luciferase Reporter Assay System (Promega Corporation) according to the manufacturer's protocol. The relative reporter activity was normalized to the ratio of firefly luciferase to Renilla luciferase.

Statistical analysis. All data are presented as the mean \pm SD. Student's unpaired t-tests (two tailed) were used for comparisons between two groups. The statistical difference of the luciferase reporter assay was evaluated using a one-way ANOVA followed by Tukey's post-hoc tests. Statistical analysis was performed using SPSS 20.0 (IBM Corp.). $\mathrm{P}<0.05$ was considered to indicate a statistically significant difference.

\section{Results}

circRNA expression profile analysis in DN mice. In order to investigate the differentially expressed circRNAs in the kidney tissue, the present study employed mouse circRNA sequencing in $d b / m$ and $d b / d b$ mice with DN. Heat maps (Fig. 1A) and volcano plots (Fig. 1B) revealed that a total of 40 circRNAs, including 18 upregulated circRNAs and 22 downregulated circRNAs, were differentially expressed, using $\mathrm{P}<0.01$ and $\mid \log 2$ (fold change) $\mid>1$ as a significant cut off criterion.

Overexpression of circRNA_0000491 in kidney tissue and high glucose-treated mouse MES13 cells. RT-qPCR was used to evaluate mmu_circ_0000712, mmu_circ_0000898, novel_ circ_0001857, circRNA_0000491 and novel_circ_0001778 levels in 3 paired kidney tissue samples (Fig. S1). It was found that the transcriptional levels of mmu_circ_0000712, novel_ circ_0001857 and novel_circ_0001778 were significantly increased in the kidney tissue of $d b / d b$ mice. Furthermore, according to the UCSC Genome Browser, mmu_circ_0000491 is produced at the Homerl gene locus, containing exons 2-5 (Fig. 1C). The results also revealed that circRNA_0000491 expression was markedly amplified in the $d b / d b$ mice (Fig. 2A). Among the upregulated circRNAs, it was noted that circRNA_0000491 was significantly increased; therefore, this circRNA was selected for further experiments. Additionally, it was observed that circ_0000491 expression was markedly upregulated in MES13 cells exposed to high glucose for 12 and $24 \mathrm{~h}$, respectively, when compared with the expression in control cells (Fig. 2B). These findings documented the high expression levels of circRNA_0000491 in $d b / d b$ mouse kidney tissue.

Knockdown of circRNA_0000491 suppresses ECM accumulation of MESI3 cells. circRNA microarray analyses identified a variety of dysregulated circRNAs in the DN mouse kidney cortex (Fig. 1A). Subsequently, the present study identified that circRNA_0000491 was expressed at significantly higher levels in the kidney tissues of the $d b / d b$ mice and it was speculated that circRNA_0000491 participated in the pathological process of ECM accumulation and renal fibrosis. In the present study, ECM accumulation and fibrosis-associated proteins levels, including E-cad, Vim, FN and $\alpha$-SMA, as well as Col. Types I, III and IV were investigated using RT-qPCR. The results suggested that the mRNA expression levels of Vim, FN, $\alpha$-SMA, Col. I, III and IV were increased, while the levels of E-cad were markedly decreased compared with 


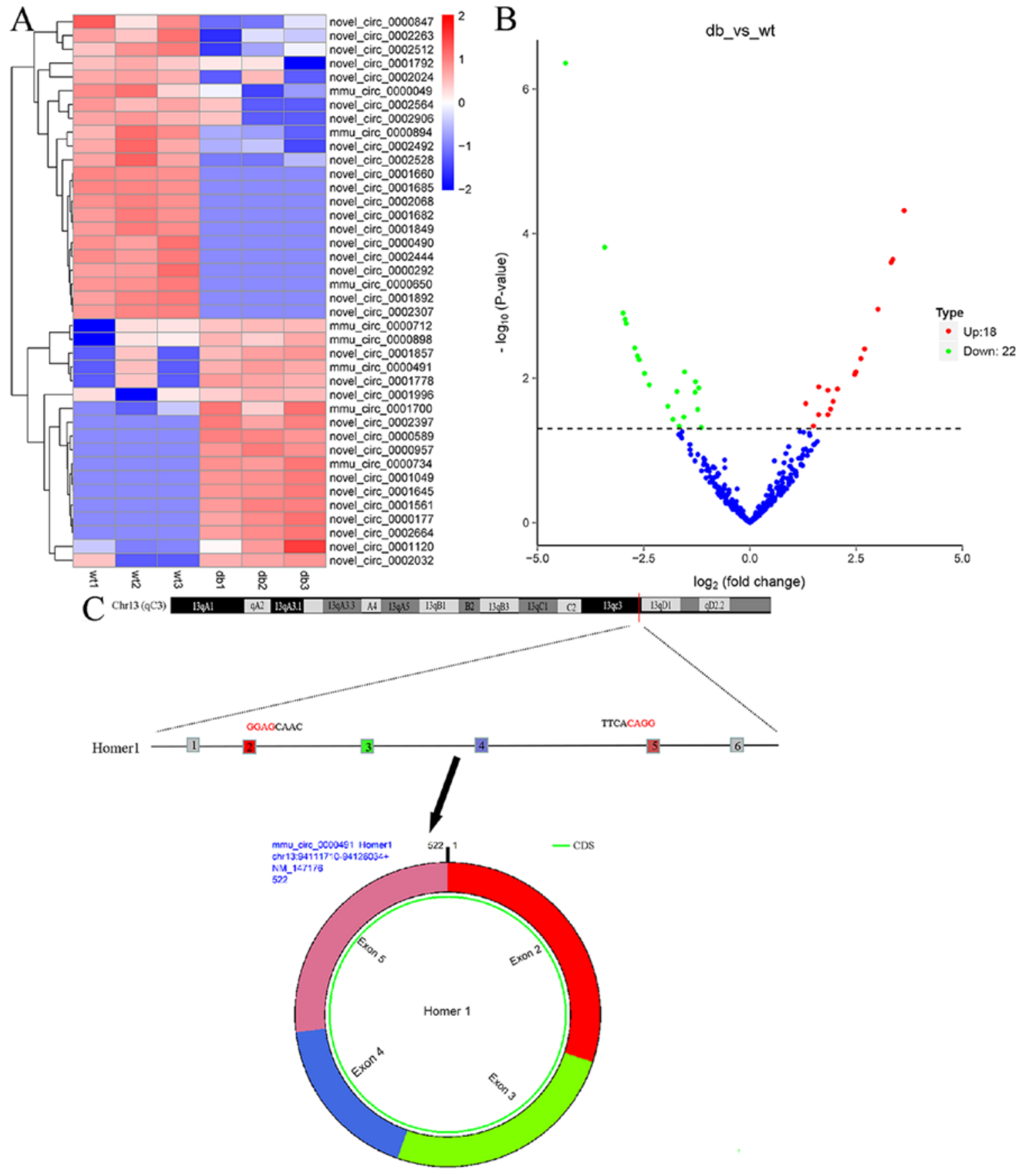

Figure 1. circRNA microarray analysis and circRNA_0000491 are upregulated in $d b / d b$ diabetic nephropathy mice kidney tissues and MES13 cells treated with high glucose. (A) Heat maps and (B) volcano plots displaying the differentially expressed circRNAs. (C) A schematic diagram of the genomic location and structure of circ_0000491. circRNA, circular RNA.

control $\mathrm{db} / \mathrm{m}$ mice and normal glucose treated MES13 cells (Fig. 3A and B). The role of circRNA_0000491 was further investigated in MES13 cells using a specific siRNA. The knockdown efficiency of si_Circ_1 and si_Circ_2 in MES13 cells was determined by RT-qPCR analysis. The results showed that the expression levels of circRNA_0000491 were significantly decreased in the si_Circ_1 group compared with that in the si_Circ_Control group. Subsequently, the present study selected si_Circ_1 as it had a more effective knockdown efficiency than si_Circ_2 for subsequent experiments (Fig. 3C). Moreover, the western blot analysis revealed that the Vim, FN, $\alpha$-SMA, Col. I, III and IV protein expression levels were suppressed and that the expression of E-cad in the si_Circ_1 treated group was observably increased compared with the si Circ_NC group in HG-treated MES13 cells (Fig. 3D-K). Taken together, these findings confirmed that circRNA_0000491 knockdown suppressed the epithelial-to-mesenchymal transition (EMT) process and fibrosis-associated protein synthesis in MES13 cells.

circRNA_0000491 sponges miR-101b in MES13 cells. In the present study, MES13 cells were transfected with miR-101b mimics or miR-101b inhibitor to achieve miR-101b expression, as illustrated in Fig. 4A. The expression of circRNA_0000491 was negatively regulated by miR-101b (Fig. 4B). The suggestion that miR-101b may be sponged by circRNA_0000491 was 

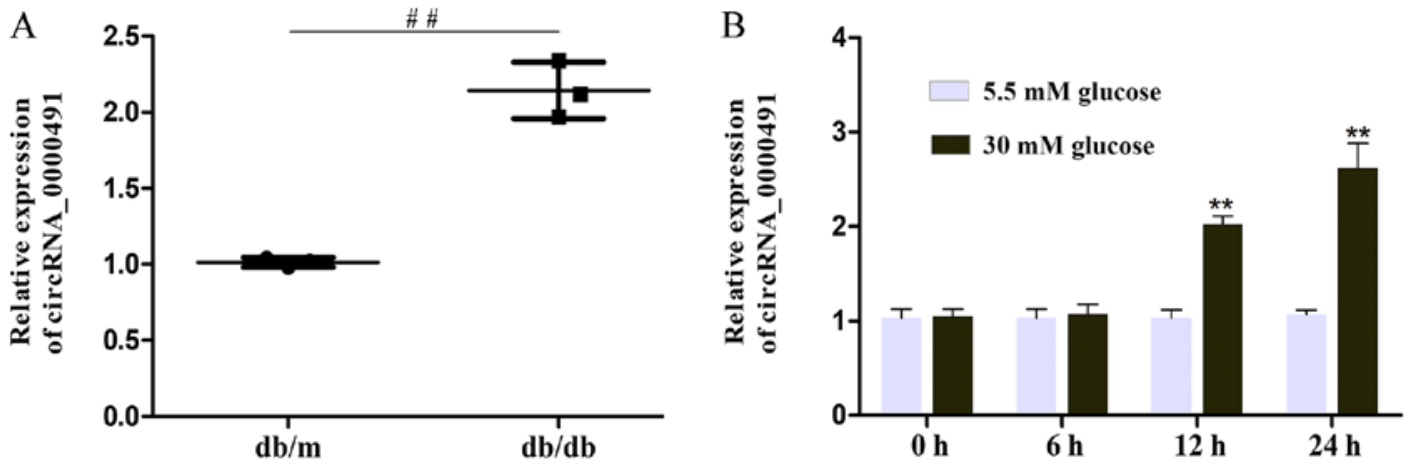

Figure 2.Expression of circRNA_0000491 in the kidney tissue of $d b / d b$ mice and high glucose-induced mouse MES13 cells.(A) Reverse transcription-quantitative PCR estimated the expression levels of circ_0000491 in diabetic nephropathy mice and (B) the high concentration glucose (30 mM)-induced MES13 cells. ${ }^{\# \#} \mathrm{P}<0.01$ vs. the $d b / m$ control group; ${ }^{* *} \mathrm{P}<0.01$ vs. the $5.5 \mathrm{mM}$ glucose treated MES13 cells group. circRNA, circular RNA.

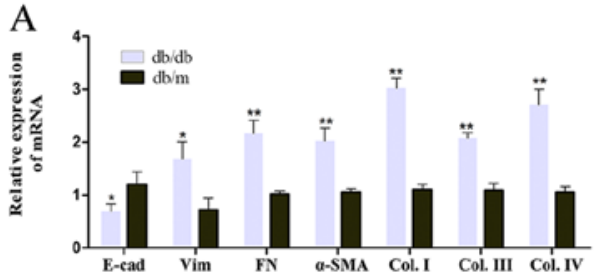

D

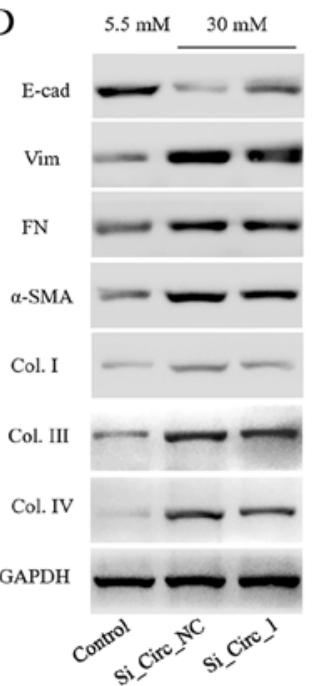

B

E
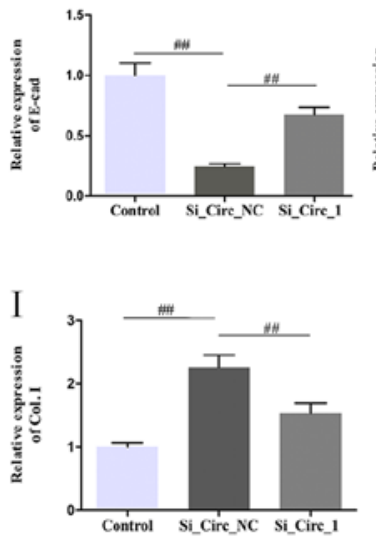

B $30 \mathrm{mM}$ ghocose

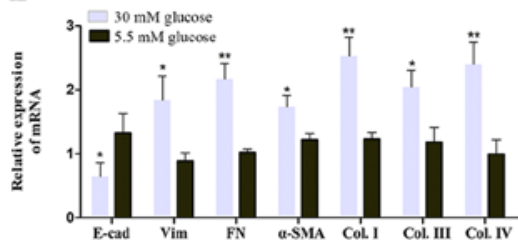

$\mathrm{C}$

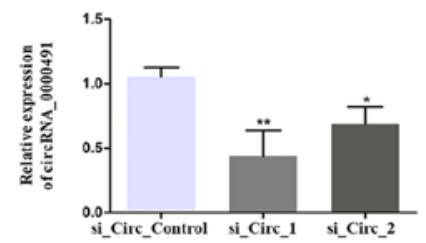

$\mathrm{H}$
F

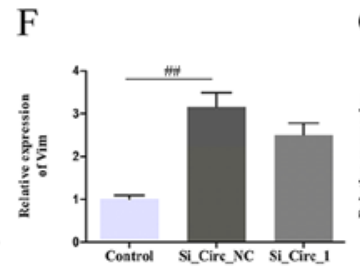

G

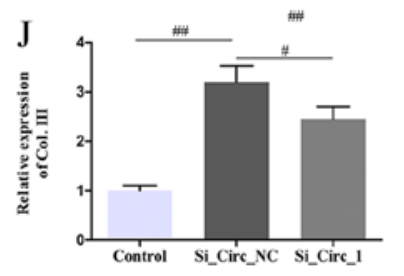

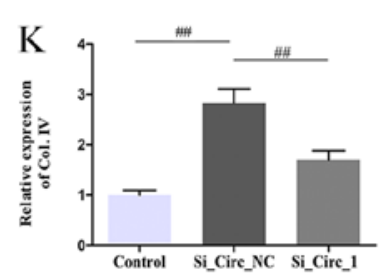

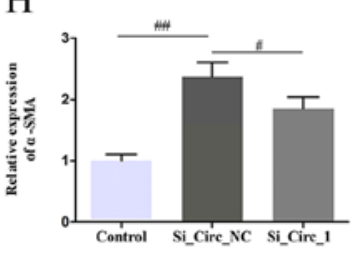

Figure 3. Circ_0000491 silencing inhibits the extracellular matrix accumulation and fibrosis-associated protein synthesis in MES13 cells. RT-qPCR revealed the expression levels of E-cad, Vim, FN, $\alpha$-SMA, Col. I, Col. III and Col. IV in (A) diabetic nephropathy mice and (B) MES13 cells treated with high glucose. (C) The expression levels of circ_0000491 in MES13 cells transfected using siRNA. (D) Western blotting showing the protein expression levels of (E) E-cad, (F) Vim, (G) FN, (H) $\alpha$-SMA, (I) Col. I, (J) Col. III and (K) Col. IV in MES13 cells with high glucose treatment and transfected with si_circ_0000491. ${ }^{*} \mathrm{P}<0.05,{ }^{* *} \mathrm{P}<0.01$ vs. the corresponding control group; ${ }^{\#} \mathrm{P}<0.05,{ }^{\# \#} \mathrm{P}<0.01$ vs. the Si_Circ_NC group. Col., collagen; circRNA, circular RNA; E-cad, E-cadherin; FN, fibronectin; si, small interfering; SMA, smooth muscle actin; Vim, vimentin.

predicted using the starBase v3.0 platform and the seeding sequences of circ_0000491 and miR-101b are presented in Fig. 4C. In order to validate whether miR-101b is the direct target of circ_0000491, a luciferase reporter assay was performed in the present study. The results revealed that miR-101b mimics significantly inhibited the luciferase activity mediated by wild-type circ_0000491-luciferase reporter, which suggested an interaction between circ_0000491 and miR-101b (Fig. 4D). In addition, the results demonstrated that miR-101b expression was significantly downregulated in DN mice and the high concentration glucose-treated group (Fig. 4E) in comparison to the control groups. Furthermore,
miRNA-101b expression was significantly increased in MES13 cells transfected with si_Circ_1 (Fig. 4F). These data suggested that circ_0000491 negatively regulates miR-101b expression by directly sponging miR-101b.

miR-101b sponges TGF $\beta R I$ in MES13 cells. After confirming that circ_0000491 sponges miR-101b in MES13 cells, it was necessary to further determine the downstream target of the circRNA_0000491/miR-101b axis. According to the prediction of the TargetScan, miRDB and PicTar databases, TGF $\beta$ RI may be a potential downstream target for miR-101b. The underlying complementary binding sequence is presented in Fig. 5A. To 
A

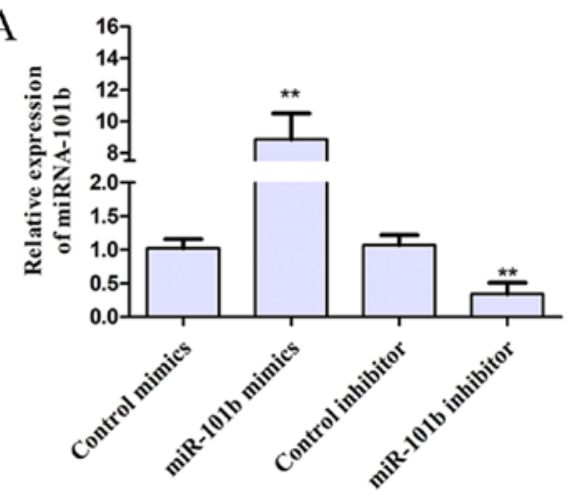

C

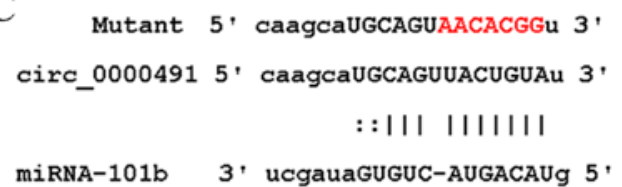

E

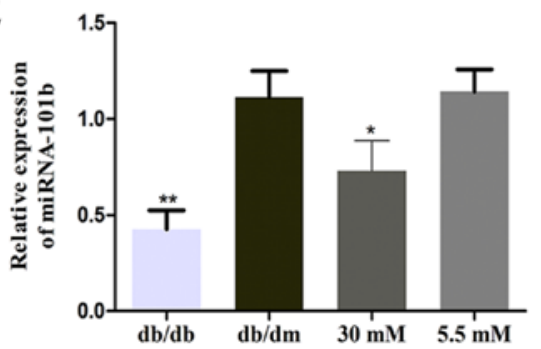

B

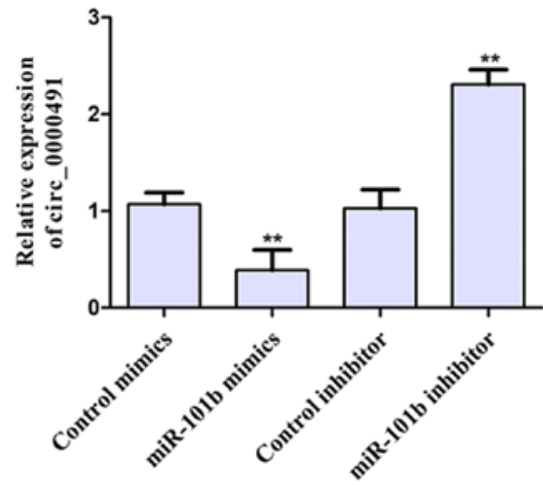

D

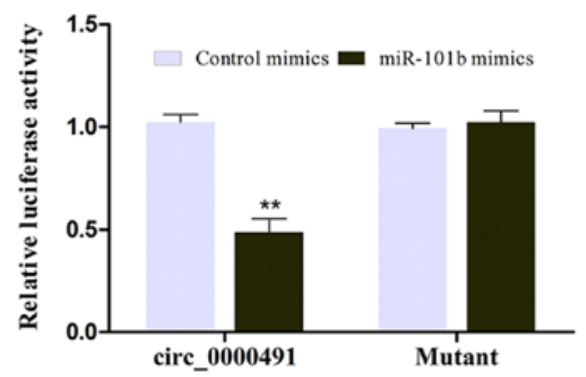

F

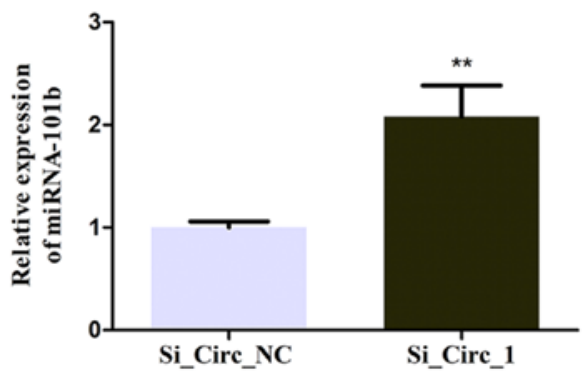

Figure 4. Circ_0000491 targets miRNA-101b. (A) The miR-101b mRNA expression levels in MES13 cells transfected with miR-101b mimics or miR-101b inhibitor was confirmed using RT-qPCR. (B) Circ_0000491 expression was examined in the transfected MES13 cells. (C) Diagram of the miR-101b putative binding sites in circ_0000491. (D) miR-101b expression in $d b / d b$ mice and high glucose treated MES13 cells was measured using RT-qPCR. (E) Luciferase reporter assay showed the binding activity of miR-101b and circRNA_0000491. (F) miR-101b mRNA expression levels in MES13 cells transfected with si_circ_0000491 was measured using RT-qPCR. ${ }^{*} \mathrm{P}<0.05,{ }^{* *} \mathrm{P}<0.01$ vs. the corresponding control group. circRNA, circular RNA; miR/miRNA, microRNA; RT-qPCR, reverse transcription-quantitative PCR; si, small interfering.

further investigate the association between miR-101b and TGF $\beta R$ I, a luciferase reporter assay was performed. It was found that the luciferase activity was significantly downregulated in wild-type TGF $\beta$ RI cells co-transfected with miR-101b mimics (Fig. 5B). Furthermore, RT-qPCR revealed that the mRNA expression levels of TGF $\beta$ RI were markedly increased in DN mice and high glucose-treated MES13 cells compared with the corresponding control groups (Fig. 5C and D). These data verified that TGF $\beta$ RI servers as the target of miR-101b.

miR $-101 b$ rescues the effects of circ_0000491 in MES13 cells by targeting TGF $\beta R I$. It was hypothesized that the miR-101b/TGFßRI axis is one-way and that circ_0000491 promotes ECM accumulation and fibrosis in DN cells. To test this hypothesis, the present study performed rescue experiments in which circ_0000491-knockdown cultures underwent transfection with an miR-101b inhibitor and scrambled control. Western blotting revealed that the protein expression of TGF $\beta 1$, TGF $\beta R I$, phosphorylated (p)Smad3, Vim, FN and $\alpha$-SMA, as well as Col. I, III and IV were all increased; however, E-cad decreased in the miR-101b inhibitor transfected cells. In addition, the cotransfection of miR-101 inhibitor and si_Circ_1 reversed the effect of circ_0000491 silencing (Fig. 6A-K). Collectively, these results suggested that circ_0000491 acts as a sponge of miR-101b, regulating high glucose-induced ECM accumulation and the expression levels of fibrosis-associated proteins in MCs by upregulating TGF $\beta$ RI and suppressing miR-101b.

\section{Discussion}

$\mathrm{DN}$ is one of the major devastating complications of diabetes mellitus (DM). Notably, 2 in every 10 patients with either 
A

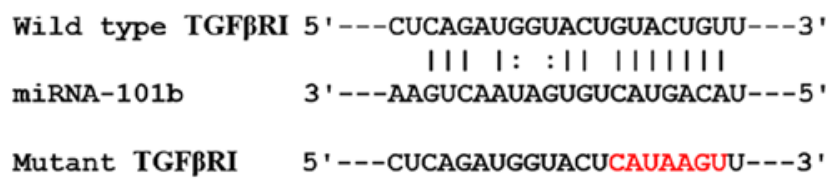

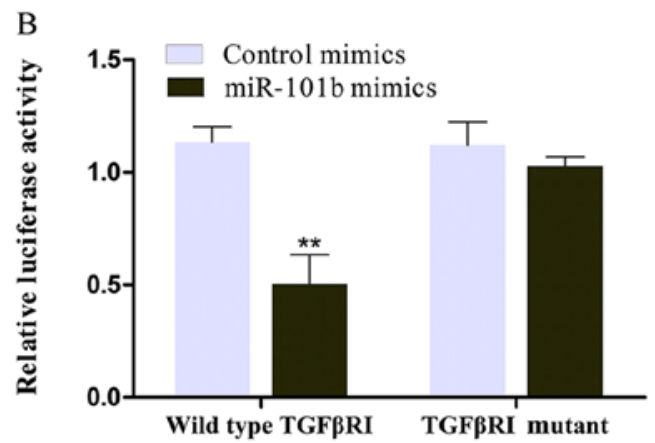

$\mathrm{D}$

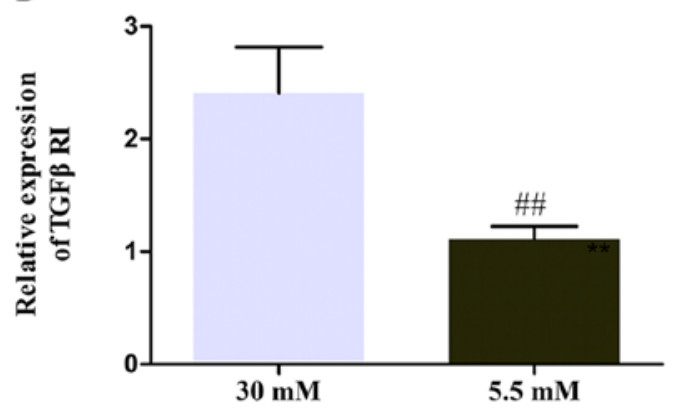

Figure 5. miRNA-101b targets TGF $\beta$ RI. (A) Diagram of the binding sites between TGF $\beta$ RI and miRNA-101b. Red text represents the mutant sequence of TGF $\beta R I$ (B) Relative luciferase activity of the wild-type and 3'-untranslated region mutant constructs of TGF $\beta$ RI co-transfected with miR-101b mimics and miRNA control. (C) The mRNA expression levels of TGF $\beta R I$ in $d b / d b$ mice. (D) The mRNA expression levels of TGF $\beta R I$ in the high glucose treated MES13 cells. ${ }^{* *} \mathrm{P}<0.01$ Control mimics + wild-type TGF $\beta$ RI group vs. miR-101b mimics + wild-type TGF $\beta$ RI group; ${ }^{\wedge} \mathrm{P}<0.05 d b / d b$ group vs. Db/dm group; ${ }^{\# \#} \mathrm{P}<0.01$ $5.5 \mathrm{mM}$ glucose treated group vs. $30 \mathrm{mM}$ glucose treated group. miR/miRNA, microRNA; TGF $\beta$ R1, TGF $\beta$ receptor 1.

type 1 or type $2 \mathrm{DM}$, will develop DN after $10-20$ years (21). The number of patients with DN was 382 million in 2013 and is estimated to reach 592 million by 2035 (22). It is evident that abundant circRNAs exist in the eukaryotic transcriptomes and accumulating evidence suggests that they may have a vital role in regulating a series of human diseases and cellular functions (23). circRNAs are closed RNA transcripts that are conserved among different biological systems. However, to the best of our knowledge, the role of circRNA in DN and the mechanisms of action by which circRNAs regulate the accumulation of the ECM and fibrosis has not been fully described. The present study aimed to investigate the circRNA microarray in the DN mice model and then clarify the underlying biological characteristics of circRNAs on the DN physiological and pathological processes.

In the present study, a subset of circRNAs were identified through the circRNA microarray analysis. Heat maps and volcano plots showed a total of 40 circRNAs were differentially expressed, including 18 upregulated circRNAs and 22 downregulated circRNAs. Among these abnormal circRNAs, the present study selected an upregulated circRNA, circRNA_0000491, and investigated the pathological phenotype associated with EMT, one of the known underlying mechanisms of DN. The present study initially assessed the potential effect of circRNA_0000491 on ECM and fibrosis-associated protein expression, then performed loss-of-function experiments through siRNA transfection. The loss-of-function experiments demonstrated that compromised circRNA_0000491 expression significantly suppressed the protein expression levels of Vim, FN and $\alpha$-SMA, as well as Col. I, III and IV, indicating that circRNA_0000491 participated in the ECM accumulation of the EMT process. This suggested that silencing its expression would be conducive to decrease the synthesis of fibrosis-associated proteins.

Accumulating studies have demonstrated that circRNAs are crucial regulators in the process of the transmission and function of genetic information, usually acting as miRNA sponges. Research on circRNAs in DM is a novel research field and further research into specific circRNA expression on the ECM is still required, despite recent advancements. For example, Wu et al (24) investigated hsa circ_0005105 expression in osteoarthritis and reported that hsa_circ_0005105 could promote ECM degradation through sponging miR-26a targeting NAMPT. Zhao et al (25) indicated that hsa_circ_0054633 may be a potential biomarker and have a diagnostic capability for pre-diabetes and type 2 DM. In CFs, circRNA_000203 specifically sponges miR-26b-5p and overexpression of circRNA_000203 may eliminate the anti-fibrotic effect of miR-26b-5p in CFs, accompanied by the suppression of collagen type III $\alpha 1$ chain (Col3a1) and $\alpha$-SMA (26). Furthermore, emerging evidence has demonstrated that circRNAs may regulate cancer cell proliferation, migration and invasion as miRNA sponge, not only in DN. For instance, Lili and Yue (27) demonstrated that significantly upregulated expression levels of hsa_circ_0007534 are present in breast cancer (BC) and knockdown of hsa_circ_0007534 inhibited BC colony formation, cell proliferation and invasion, as well as strengthening 

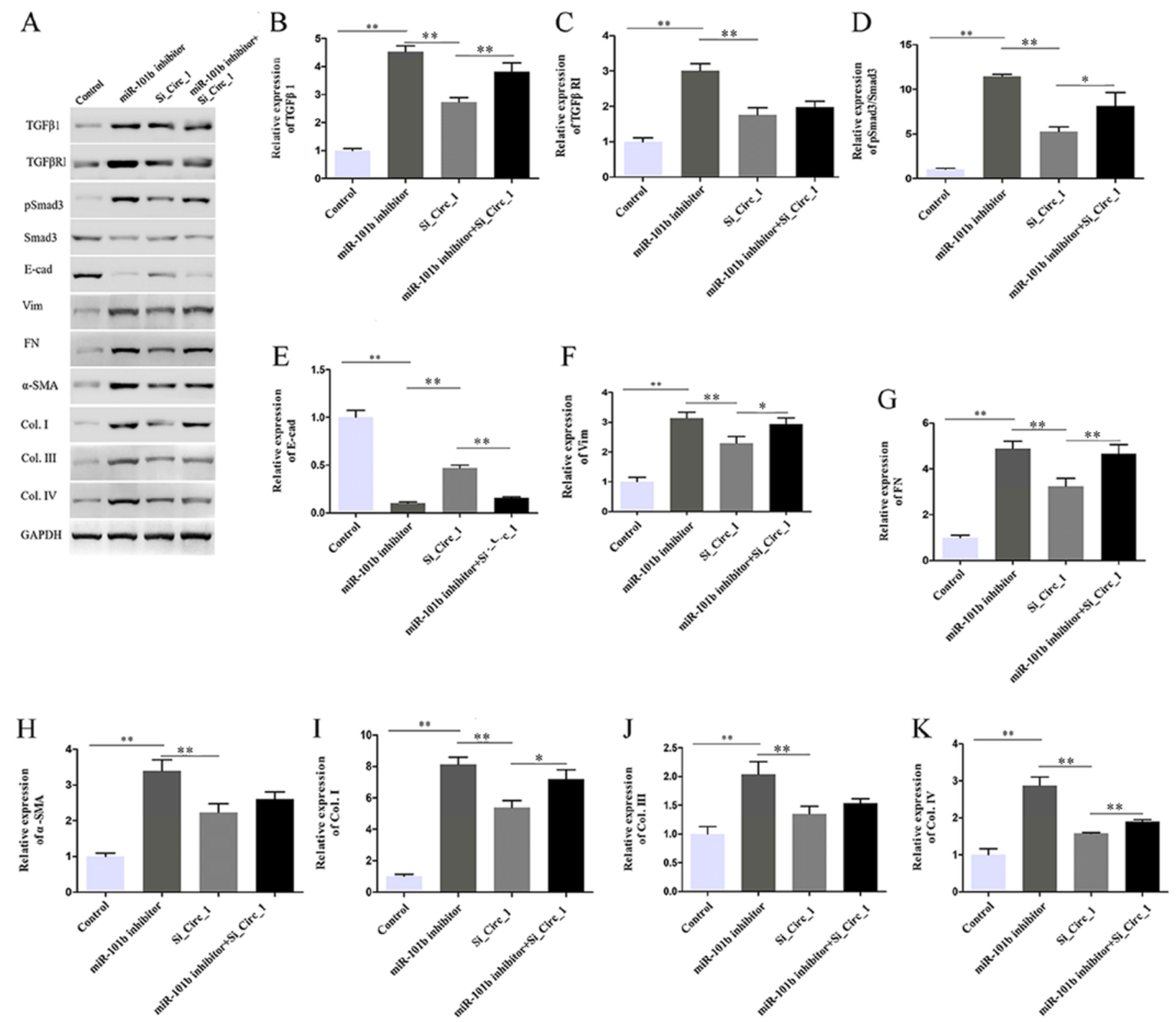

Figure 6. Effect of circ_0000491 and miRNA-101b inhibition on extracellular matrix and fibrosis-associated protein expression via targeting TGFßRI. (A) Western blotting showed that miR-101b inhibitor reversed the effect of si_circRNA_0000491 on the protein expression levels of (B) TGF $\beta 1$, (C) TGF $3 R I$, (D) pSmad3/Smad3, (E) E-cad, (F) Vim, (G) FN, (H) $\alpha$-SMA, (I) Col. I, (J) Col. III and (K) Col. IV. " P<0.05, ** P<0.01. Col., collagen; circRNA, circular RNA; E-cad, E-cadherin; FN, fibronectin; miR/miRNA, microRNA; p, phosphorylated; si, small interfering; SMA, smooth muscle actin; TGF $\beta$ R1, TGF $\beta$ receptor 1; Vim, vimentin.

apoptosis in BC cells by acting as a miR-593 sponge to raise mucin 19, oligomeric (MUC19) expression levels. In gastric cancer, the knockdown of hsa_circ_0001368 results in accelerated tumor growth in vivo and may act as a ceRNA to sponge miR-6506-5p and play a tumor-suppressive role (28). Liu et al (29) suggested that circ_0080425 functions as sponge, harboring miR-24-3p, which inhibits cell proliferation and fibrosis in DN by targeting fibroblast growth factor 11. Furthermore, Yao et al (30) demonstrated that circ_0000285 aggravates podocyte injury through sponging miR-654-3p and activating MAPK6 in DN. Taken together, these results provide novel insights into circRNAs and add to the growing amount of evidence that circRNAs can sequester miRNAs.

Mechanistically, in the present study, the interaction between miR-101b and circRNA_0000491 was predicted using bioinformatics analysis, and was confirmed by the luciferase report assay. Thus, circRNA_0000491 may act as an endogenous miR-101b sponge to promote ECM degradation. Additionally, the target genes of miR-101b were predicted using bioinformatics analysis. Subsequently, dual-luciferase reporter assays further confirmed that TGF $\beta$ RI serves as the target gene of miR- $101 \mathrm{~b}$. TGF- $\beta 1 /$ Smad signaling is critical in the process of EMT. Dysregulation of the signaling pathway can contribute to abnormal ECM deposition, causing extensive kidney fibrosis $(31,32)$. In this pathway, TGF- $\beta 1$ exerts biological effects through binding to type II $\beta-\beta$ receptor and subsequently recruits and activates TGF $\beta R I$, then the activated TGFßR1 phosphorylates Smad2/3 (33,34). Previous studies have demonstrated that inhibiting TGF $\beta$ RI significantly improves various disease, including pulmonary fibrosis (35), hypertensive nephropathy (36) and tubulo-interstitial 
fibrosis (37), suggesting that inhibiting TGF $\beta$ RI may be a promising anti-fibrotic therapeutic strategy for DN. These findings are consistent with the current study where it was found that circRNA_0000491 knockdown inhibited TGF $\beta 1$, TGF $\beta R I$ and pSmad3 protein expression levels $(34,36)$.

In conclusion, the high expression levels of circRNA_0000491 in $d b / d b$ mice and high concentration glucose-induced MCs was negatively correlated with miR-101b expression. Furthermore, the results from the present study indicated that the circRNA_0000491/miR-101b/TGF $\beta$ RI axis may regulate ECM and fibrosis-associated protein synthesis of DN. Therefore, circRNA_0000491 may be considered as a DN-promoting gene and may represent a novel insight for the treatment of DN.

\section{Acknowledgements}

Not applicable.

\section{Funding}

The present study was supported by the National Natural Science Foundation of China (grant nos. 81774217 and 81273623), Zhejiang Traditional Chinese Medicine Administration (grant nos. 2017ZKL016 and 2019ZB096) and the Science and Technology Commission of Hangzhou (grant no. 20150733Q42).

\section{Availability of data and materials}

The datasets analyzed/generated during the present study are available from the corresponding author upon reasonable request.

\section{Authors' contributions}

XM, JWC, DYZ and YBH conceived and designed the present study. XM, KL and LJC performed the experimental procedures. KL, LJC and DZ analyzed the data. XM, JWC and YBH drafted the manuscript. All authors read and approved the final manuscript.

\section{Ethics approval and consent to participate}

The present study was approved by the Animal Care and Welfare Committee of Zhejiang Chinese Medical University.

\section{Patient consent for publication}

Not applicable.

\section{Competing interests}

The authors declare that they have no competing interests.

\section{References}

1. Chen $\mathrm{B}, \mathrm{Li} \mathrm{Y}$, Liu $\mathrm{Y}$ and $\mathrm{Xu} \mathrm{Z}$ : circLRP6 regulates high glucose-induced proliferation, oxidative stress, ECM accumulation, and inflammation in mesangial cells. J Cell Physiol 234: 21249-21259, 2019.
2. Chen C, Gong W, Li C, Xiong F, Wang S, Huang J, Wang Y, Chen Z, Chen Q, Liu P, et al: Sphingosine kinase 1 mediates AGEs-induced fibronectin upregulation in diabetic nephropathy. Oncotarget 8: 78660-78676, 2017.

3. Wang Y, He Z, Yang Q and Zhou G: XBP1 inhibits mesangial cell apoptosis in response to oxidative stress via the PTEN/AKT pathway in diabetic nephropathy. FEBS Open Bio 9: 1249-1258, 2019.

4. Chen Z, Chen Q, Huang J, Gong W, Zou Y, Zhang L, Liu P and Huang $\mathrm{H}$ : $\mathrm{CK} 2 \alpha$ promotes advanced glycation end products-induced expressions of fibronectin and intercellular adhesion molecule-1 via activating MRTF-A in glomerular mesangial cells. Biochem Pharmacol 148: 41-51, 2018.

5. Hu W, Han Q, Zhao L and Wang L: Circular RNA circRNA_15698 aggravates the extracellular matrix of diabetic nephropathy mesangial cells via miR-185/TGF- $\beta 1$. J Cell Physiol 234: 1469-1476, 2019.

6. Zhang P, Sun Y, Peng R, Chen W, Fu X, Zhang L, Peng H and Zhang Z: Long non-coding RNA Rpph1 promotes inflammation and proliferation of mesangial cells in diabetic nephropathy via an interaction with Gal-3. Cell Death Dis 10: 526, 2019.

7. Zha F, Qu X, Tang B, Li J, Wang Y, Zheng P, Ji T, Zhu C and Bai S: Long non-coding RNA MEG3 promotes fibrosis and inflammatory response in diabetic nephropathy via miR-181a/Egr-1/TLR4 axis. Aging (Albany NY) 11: 3716-3730, 2019.

8. Ebbesen KK, Hansen TB and Kjems J: Insights into circular RNA biology. RNA Biol 14: 1035-1045, 2017.

9. Wang T, Wang X, Du Q, Wu N, Liu X, Chen Y and Wang X: The circRNA circP4HB promotes NSCLC aggressiveness and metastasis by sponging miR-133a-5p. Biochem Biophys Res Commun 513: 904-911, 2019.

10. Zhou B and Yu JW: A novel identified circular RNA, circRNA_010567, promotes myocardial fibrosis via suppressing miR-141 by targeting TGF- $\beta 1$. Biochem Biophys Res Commun 487: 769-775, 2017.

11. Wang W, Feng J, Zhou H and Li Q: Circ_0123996 promotes cell proliferation and fibrosisin mouse mesangial cells through sponging miR-149-5p and inducing Bach1 expression. Gene 761: $144971,2020$.

12. Li S, Pei Y, Wang W, Liu F, Zheng K and Zhang X: Circular RNA 0001785 regulates the pathogenesis of osteosarcoma as a ceRNA by sponging miR-1200 to upregulate HOXB2. Cell Cycle 18: 1281-1291, 2019.

13. Ni H, Li W, Zhuge Y, Xu S, Wang Y, Chen Y, Shen G and Wang F: Inhibition of circHIPK3 prevents angiotensin II-induced cardiac fibrosis by sponging miR-29b-3p. Int J Cardiol 292: 188-196, 2019.

14. Memczak S, Jens M, Elefsinioti A, Torti F, Krueger J, Rybak A Maier L, Mackowiak SD, Gregersen LH, Munschauer M, et al: Circular RNAs are a large class of animal RNAs with regulatory potency. Nature 495: 333-338, 2013.

15. Gao Y, Zhang J and Zhao F: Circular RNA identification based on multiple seed matching. Brief Bioinform 19: 803-810, 2018.

16. Kent WJ, Sugnet CW, Furey TS, Roskin KM, Pringle TH, Zahler AM and Haussler D: The human genome browser at UCSC. Genome Res 12: 996-1006, 2002.

17. Livak KJ and Schmittgen TD: Analysis of relative gene expression data using real-time quantitative PCR and the 2(-Delta Delta C(T)) method. Methods 25: 402-408, 2001.

18. Li JH, Liu S, Zhou H, Qu LH and Yang JH: StarBase v2.0: Decoding miRNA-ceRNA, miRNA-ncRNA and protein-RNA interaction networks from large-scale CLIP-Seq data. Nucleic Acids Res 42: D92-D97, 2014.

19. Agarwal V, Bell GW, Nam JW and Bartel DP: Predicting effective microRNA target sites in mammalian mRNAs. Elife 4: e05005, 2015 .

20. Chen Y and Wang X: miRDB: An online database for prediction of functional microRNA targets. Nucleic Acids Res 48: D127-D131, 2020.

21. He M, Wang J, Yin Z, Zhao Y, Hou H, Fan J, Li H, Wen Z, Tang J, Wang Y, et al: MiR-320a induces diabetic nephropathy via inhibiting MafB. Aging (Albany NY) 11: 3055-3079, 2019.

22. Leon BM and Maddox TM: Diabetes and cardiovascular disease: Epidemiology, biological mechanisms, treatment recommendations and future research. World J Diabetes 6: 1246-1258, 2015.

23. Yu CY, Li TC, Wu YY, Yeh CH, Chiang W, Chuang CY and Kuo HC: The circular RNA circBIRC6 participates in the molecular circuitry controlling human pluripotency. Nat Commun 8: 1149, 2017.

24. Wu Y, Zhang Y, Zhang Y and Wang JJ: CircRNA hsa circ_0005105 upregulates NAMPT expression and promotes chondrocyte extracellular matrix degradation by sponging miR-26a. Cell Biol Int 41: 1283-1289, 2017. 
25. Zhao Z, Li X, Jian D, Hao P, Rao L and Li M: Hsa circ 0054633 in peripheral blood can be used as a diagnostic biomarker of pre-diabetes and type 2 diabetes mellitus. Acta Diabetol 54: 237-245, 2017.

26. Tang CM, Zhang M, Huang L, Hu ZQ, Zhu JN, Xiao Z, Zhang Z, Lin QX, Zheng XL, Yang M, et al: CircRNA_000203 enhances the expression of fibrosis-associated genes by derepressing targets of miR-26b-5p, Col1a2 and CTGF, in cardiac fibroblasts. Sci Rep 7: 40342, 2017.

27. Lili S and Yue X: Downregulation of hsa_circ_0007534 suppresses breast cancer cell proliferation and invasion by targeting miR-593/MUC19 signal pathway. Biochem Biophys Res Commun 503: 2603-2610, 2018.

28. Lu J, Zhang PY, Li P, Xie JW, Wang JB, Lin JX, Chen QY, Cao LL, Huang CM and Zheng CH: Circular RNA hsa_circ_0001368 suppresses the progression of gastric cancer by regulating miR-6506-5p/FOXO3 axis. Biochem Biophys Res Commun 512: 29-33, 2019.

29. Liu H, Wang X, Wang ZY and Li L: Circ_0080425 inhibits cell proliferation and fibrosis in diabetic nephropathy via sponging miR-24-3p and targeting fibroblast growth factor 11 . J Cell Physiol 235: 4520-4529, 2020.

30. Yao T, Zha D, Hu C and Wu X: Circ 0000285 promotes podocyte injury through sponging miR-654-3p and activating MAPK6 in diabetic nephropathy. Gene 747: 144661, 2020.

31. Sutariya B, Jhonsa D and Saraf MN: TGF- $\beta$ : The connecting link between nephropathy and fibrosis. Immunopharmacol Immunotoxicol 38: 39-49, 2016.
32. Wang Y, Liu L, Peng W, Liu H, Liang L, Zhang X, Mao Y, Zhou X, Shi M, Xiao Y, et al: Ski-related novel protein suppresses the development of diabetic nephropathy by modulating transforming growth factor- $\beta$ signaling and microRNA-21 expression. J Cell Physiol 234: 17925-17936, 2019.

33. Tu X, Zhang H, Zhang J, Zhao S, Zheng X, Zhang Z, Zhu J, Chen J, Dong L, Zang Y and Zhang J: MicroRNA-101 suppresses liver fibrosis by targeting the TGF $\beta$ signalling pathway. J Pathol 234: 46-59, 2014.

34. Derynck R and Zhang YE: Smad-dependent and Smad-independent pathways in TGF-beta family signalling. Nature 425: 577-584, 2003.

35. Li L, Ma L, Wang D, Jia H, Yu M, Gu Y, Shang H and Zou Z: Design and synthesis of matrine derivatives as novel anti-pulmonary fibrotic agents via repression of the TGF $\beta / \mathrm{smad}$ pathway. Molecules 24: 1108, 2019.

36. Ding H, Zhou Y and Huang H: MiR-101a ameliorates AngII-mediated hypertensive nephropathy by blockade of TGF $\beta / S m a d 3$ and NF- $\kappa$ B signalling in a mouse model of hypertension. Clin Exp Pharmacol Physiol 46: 246-254, 2019.

37. Wang M, Chen DQ, Wang MC, Chen H, Chen L, Liu D, Zhao H and Zhao YY: Poricoic acid ZA, a novel RAS inhibitor, attenuates tubulo-interstitial fibrosis and podocyte injury by inhibiting TGF- $\beta$ /Smad signaling pathway. Phytomedicine 36: 243-253, 2017.

This work is licensed under a Creative Commons Attribution-NonCommercial-NoDerivatives 4.0 International (CC BY-NC-ND 4.0) License. 